\title{
Development of n-DoF preloaded structures for impact mitigation in cobots
}

\author{
Authors: S. SERIANI ${ }^{*}{ }^{1}$, P. GAllina $^{1}$, L. SCALERA ${ }^{2}$, V. Lughi ${ }^{1}$ \\ 1. University of Trieste, Department of Architecture and Engineering \\ 2. University of Udine, Politechnical Department of Engineering and Architecture \\ * corresponding author: S. Seriani, sseriani@units.it, Via A. Valerio 10, 34127, Trieste (TS), Italy
}

\begin{abstract}
A core issue in collaborative robotics is that of impact mitigation, especially when collisions happen with operators. Passively compliant structures can be used as the frame of the cobot, although, usually, they are implemented by means of a single DoF. However, nDoF preloaded structures offer a number of advantages, in terms of flexibility in designing their behavior. In this work we propose a comprehensive framework for classifying n-DoF preloaded structures, including 1-, 2-, and 3-dimensional arrays. Furthermore, we investigate the implications of the peculiar behavior of these structures - which present sharp stiff-to-compliant transitions at design-determined load thresholds - on impact mitigation. To this regard, an analytical n-DoF dynamic model was developed and numerically implemented. A prototype of a 10-DoF structure was tested under static and impact loads, showing a very good agreement with the model. Future developments will see the application of $\mathrm{n}-\mathrm{DoF}$ preloaded structures to impactmitigation on cobots and in the field of mobile robots, as well as to the field of novel architected materials.
\end{abstract}

\section{INTRODUCTION}

In the modern industrial robotics, collaborative robots, often called cobots, are ever more present and are transforming many processes in the industry. Unlike traditional robots which have to be confined in a protected environment, these systems can operate in close proximity to workers, even sharing their workspace [1]. As such, cobots must be able either to avoid or to mitigate impact damage during the unavoidable collisions that may occur with an obstacle or operator. The work presented in this article originates from research done primarily in the field of collaborative and "soft" robotics and that of impact energy absorption, especially in those aspects which exploit preload. The work by Yamada et al. [2] can be considered seminal in the assessment of human-robot collisions and the active mitigation of their effects. In 2000, Lim et al. introduce the concept of passive safeguarding via viscoelastic covers and movable bases[3]. In 2005, Yoon et al. propose an early passive rotary compliant joint for use in cobots based on magnetorheological fluids [4]. In general, active protection tries to limit collisions either before or immediately upon contact [2]; conversely, passive protection generally involves compliant systems which act during collision. In order to avoid damage and accidents with workers, during collisions a threshold can be imposed to the maximum impact force by using compliant systems [4].

A mechanism referred to as "safe link" was illustrated by Park et al. in numerous aspects [5]-[8]; this introduced the concept that a cobot should take advantage of strongly non-linear stiffness, to keep positional accuracy high, while allowing for collision mitigation through compliance after a certain force threshold is reached. In 2015 López-Martínez et al. proposed a simple device [9] to implement this non-linearity by taking advantage of a preloaded compression spring; this effectively splits the elastic behaviour of the link into two areas, stiff or compliant, based on the force exerted on the end-effector; additionally, ways to actively vary the force threshold are illustrated. In order to limit collision torques in joints, in 2016, Medina et al. showed a device that implements a multiple-phase elastic field, which they called Mechanism of Multiple Joint Stiffness (MMJS) [10]. The subject of human-robot collision was investigated in several works, both experimentally and numerically, starting from Yamada et al. work [2], until recent years. In particular, Lauzier and Gosselin proposed a collision model for the reduction of the maximum contact force during blunt collisions between a robot and a human [11], whereas Park et al. presented a novel robot-human collision model consisting of a 6-degree-of-freedom mass-spring-damper system for impact analysis [12]. Other examples are given by the work of López-Martínez et al., who studied a flexible multibody model of a safety robot arm [13] and Courreges et al. who presented a new methodological approach to define a mechanical model of forcedeformation response for biologically inspired safety mechanisms [14]. The concept of variable stiffness for cobots operating in unknown environments with the risk of hitting unforeseen rigid obstacles or humans has also been discussed in the works of Wolf et al. [15], Tonietti et al. [16] and Mathijssen et al. [17].

The use of preload to increase the rigidity of structures can be found in several areas of structural engineering, e.g. in the design of large telescopes [18], in space docking systems [19] in variable-stiffness mechanisms [20] and even in pipe joints [21].

The influence of preload in energy-wise impact mitigation has seen limited interest outside of some very narrow areas. One is represented by suspension systems, present in the majority of locomotion systems, from automotive, [22], [23] to railways [24]. 
Up to this point, all cited research was concerned mainly on illustrating single-d.o.f. systems, such as active or passive joints, link structures etc. Little research has been directed towards providing a framework for general and multiple-d.o.f. preloaded structures; some exceptions are the work of Pashkevich et al., who produce a stiffness model for multiple d.o.f. passive elastic joints [25]. However, these appear to consider only perfect, linear, i.e. non-preloaded, elastic joints.

A very comprehensive review on flexible or compliant manipulators was produced by Dwivedy et al. [26]; an alternative review was published by Shabana [27]. Recently, the dynamics of the flexible multibody systems involved in this field was addressed by Bauchau et al. [28] and by Boscariol et al. [29].

Interesting examples of applications involving humanrobot interactions that require actuators with a nonclassical stiff behavior can be found in the works of Vanderborght et al. [30], where a review on variable impedance actuators is presented, and Lauzier and Gosselin [31], who proposed a series of clutch actuators for safe physical interaction between human operator and robot.
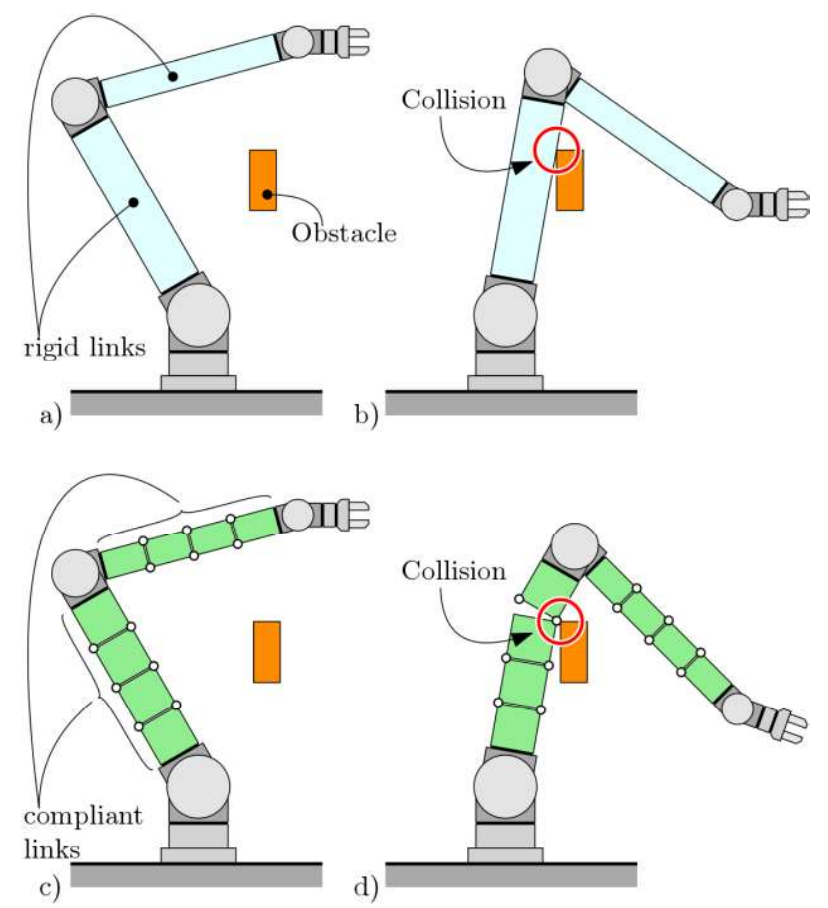

Fig. 1. Behaviour of cobots during a collision. In a) a rigid-link cobot is shown in its initial position with respect to an obstacle; in b) the collision between the two is shown. In c) a cobot with compliant links is shown, and in d) the collision is shown to cause an elastic deformation of the first link.

In this paper we propose an n-degrees of freedom model for passively compliant links that could be implemented in cobots, as illustrated in Fig. 1; this would allow compliance for the whole structure of the robot.
Furthermore, we validate the model with a experimental prototype. This is an advancement compared to state-ofthe-art mechanisms, which tend to implement the compliance either in the joints or in a specific part of the links.

The article is organized as follows: in Section 2 we define a comprehensive framework to give some coherence into preloaded mechanisms and structures with special emphasis on robotics. Section 3 is where a general model is described for $\mathrm{n}$-dof preloaded planar beam-type structures; comparative numerical and experimental validation with the prototype is also presented. Finally in Section 4 we conclude, by summarizing the present situation and giving some insight into the future works which are planned, along with potential new approaches.

\section{A FRAMEWORK FOR MULTIPLE D.O.F. PRELOADED MECHANISMS}

In order to give the most general discussion on these impact mitigation mechanisms, it is useful to define a common framework. In the following sections the effects of preload non-linearity on collisions management are presented. Furthermore, the definition is given of general n-dof structures that take advantage of preload; additionally, extension to $2 \mathrm{D}$ and $3 \mathrm{D}$ implementation is discussed.

\subsection{The effect of preload on impact mitigation}

In mechanics, elasticity is often modeled as a linear relation $F=k x$ between force $F$ and deformation, where $k$ is called elastic coefficient. However, as mentioned in the introduction, in cobots a high stiffness is required up to a certain load, and past that, compliancy: this is a nonlinear behavior.

Indeed, the simplest "soft" linearly elastic compliant link or actuator would have very poor performances, causing oscillations and poor dynamic stability during normal operation. A very simple unidimensional representation of this type of mechanism is in Fig. 2a; from the plot in Fig. $2 \mathrm{c}$, it can be seen that a non-negligible deformation $x$ can happen even with small forces. In order to avoid or limit the deformation of the structure when the applied forces are below a pre-determined level, a non-linear behavior could be implemented; one example is the Safe-link mechanism [5]. However, the most fundamental way to do this is by applying preload to the relevant elastic components. A simple implementation can be seen from the schematics in Fig. 2b and from the plot in Fig. 2d. This arrangement effectively splits the elastic field in two areas, based on loading conditions: one where the system is as rigid as the material which is used for the structure (elastic coefficient $k_{1}$ ) and the other where rigidity is given by the 
elastic components (coefficient $k_{2}$ ); typically $k_{1} \gg k_{2}$. In the plot, $F_{p}$ is the preload force: indeed, if the applied force $F \leq F_{P}$, then, in this case, the resulting deformation $x$ can be less substantial.

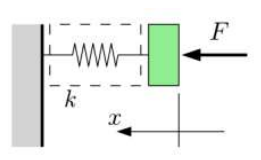

a)

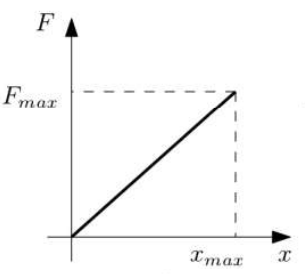

c)

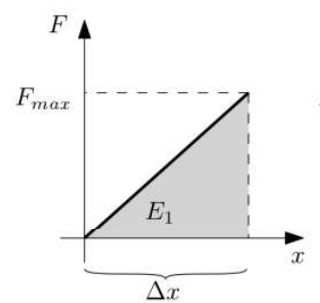

e)

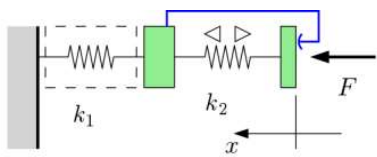

b)

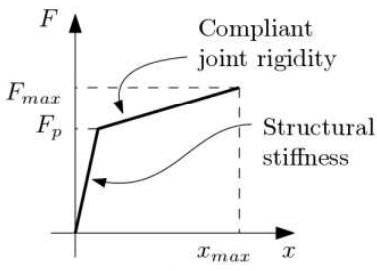

d)

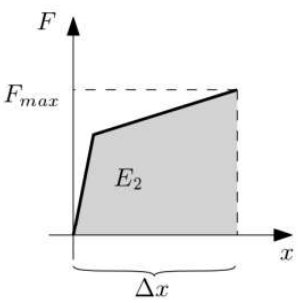

f)
Fig. 2. Difference between linear and preloaded elastic structures. In a) a simple linearly elastic structure is shown, while in b) a preloaded elastic mechanism is visible in series to the structure; preload is defined by the pair of outward-looking triangles, indicating a compressed spring. Respectively, in c) and d) the force-displacement relations between linear and preloaded structures are illustrated, whereas in e) and f) the stored elastic energies $E_{1,2}$ are represented for the two cases.

The field which historically has seen wider application of preloaded mechanisms is that of wheeled locomotion systems. When traversing an arbitrarily rough terrain, the structure experiences continuous impacts due to the unevenness of the surface [22]. Suspensions are used to mitigate the effects of these loading conditions, and must be designed to limit the impact-induced maximum force $F_{\text {max }}$ exerted on the suspended mass of the vehicle. At the same time, the maximum deflection must be limited, often to keep the mechanism compact. In energy terms, in order to optimize impact mitigation, the energy absorbed by the system should be maximized, while keeping the deflection and maximum force below a certain threshold. The diagram in Fig. 2d clearly shows that, given these prerequisites, the application of preload increases the storage of impact energy. Let us consider the mechanism in Fig. 2a, which represents a simplified robot, and the one in Fig. 2b, representing a robot with a compliant mechanism (a preloaded spring). If $E_{1}=\Delta x F_{\max } / 2$ and (with some small approximation) $E_{2} \cong \Delta x\left(F_{p}+F_{\text {max }}\right) / 2$ represent the energy stored in the non-preloaded and preloaded case respectively, it is clearly true that $E_{2}>E_{1}$ provided that $F_{p}>0$ and $k_{1}>k_{2}$.

\subsection{A comprehensive framework}

As discussed in the above section, the application of

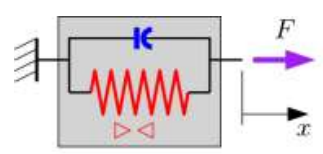

a)

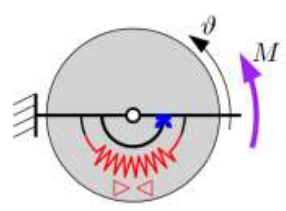

d)

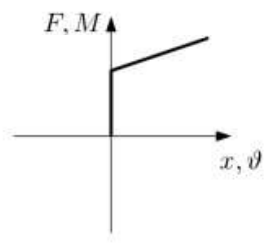

g)

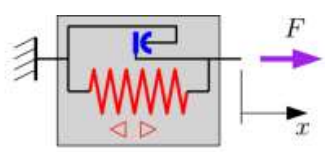

b)

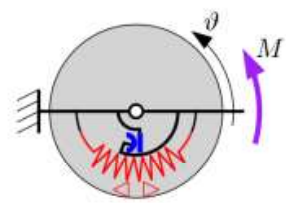

e)

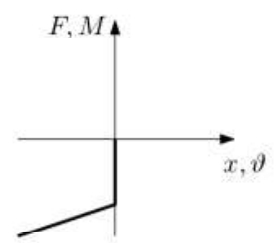

h)

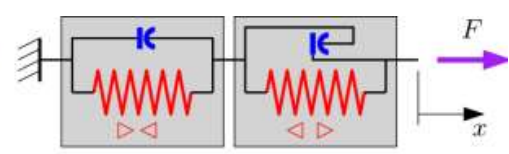

c)

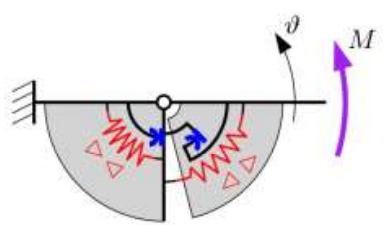

f)

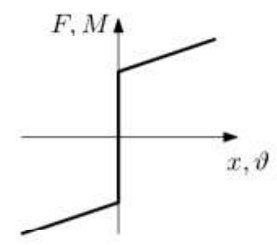

i)

Fig. 3. Definitions of the fundamental preloaded elements and their elementary combinations, along with an illustration of the nominal elastic behavior. In a) a translational traction element is shown. The mechanical limit is shown in blue, where the spring preload direction is indicated by the inward facing triangles, meaning that a traction preload is present. In b) the compression counterpart is shown, in this case the preload is compressive; in c) the combination of a) and b) is visible. In d), e) and f) the same is illustrated for the rotational case. The plots in g), h), i) show the elastic law for the solutions above each one. 
preload on structures, intended in the most general term, causes a splitting of the elastic field. Structures thus configured act differently based on the magnitude of forces acting upon them.

Given the broadness of possible configurations, however, it is useful to provide some insight into a possible classification framework. The fundamental preloaded element is that of a spring associated with a mechanical limit in its travel, e.g. as shown in Fig. 3a. The mechanical limit shown in the figure (in blue) constrains the motion of the spring, thus limiting the travel when preload is applied. This mechanism can be applied to rotational systems as well, as Fig. 3d shows. Several combinations of the fundamental elements are possible. For example, in Fig. $3 \mathrm{c}$, a traction preloaded element (Fig. 3a) is placed in series with a compression preloaded element (Fig. 3b): this causes the S-shaped behaviour visible in Fig. 3i, where motion of the free end of the combined system is possible in either direction, albeit with a non-linear elastic relation. In practice, the system appears non-compliant as long as the input force $F$ does not exceed the preload force $F_{p}$. Considering the peculiar shape that the elastic field takes, we decided to call these assemblies "S-structures".

It is now possible to extend this concept both by defining combinations of linear and rotational elementary elements, and by defining preloaded assemblies or arrays that span 2 and even 3 dimensions. In order to present some possible architectures, a classification matrix is shown in Fig. 4.

The elastic relations between the various elements can be very complex, given the interrelations between the elements in 2D planar or 3D bulk structures. The description of a complete n-D model to represent preloaded structures is out of scope for this article; it is however possible to argue that, since a closed form solution is unlikely to exist, a numeric approach would be the only viable route.

Collaborative robots can benefit from these structures; starting from simple monodimensional compliant links, all the way to $2 \mathrm{D}$ or even $3 \mathrm{D}$ "soft" arrays that act as the structure of the links, providing, in case of impact, compliance in all directions and in most parts of the system. A practical example is that of a gripper: the "fingers" can be built as a 2D array of small preloaded elements; this gives a certain structural stiffness to the elements, thus granting precise prehension, at the same time allowing flexibility in case the gripped objects are forcefully dislodged.

3 A MODEL FOR MULTIPLE-D.o.F. SSTRUCTURES

As reported in the Introduction, research in the field of

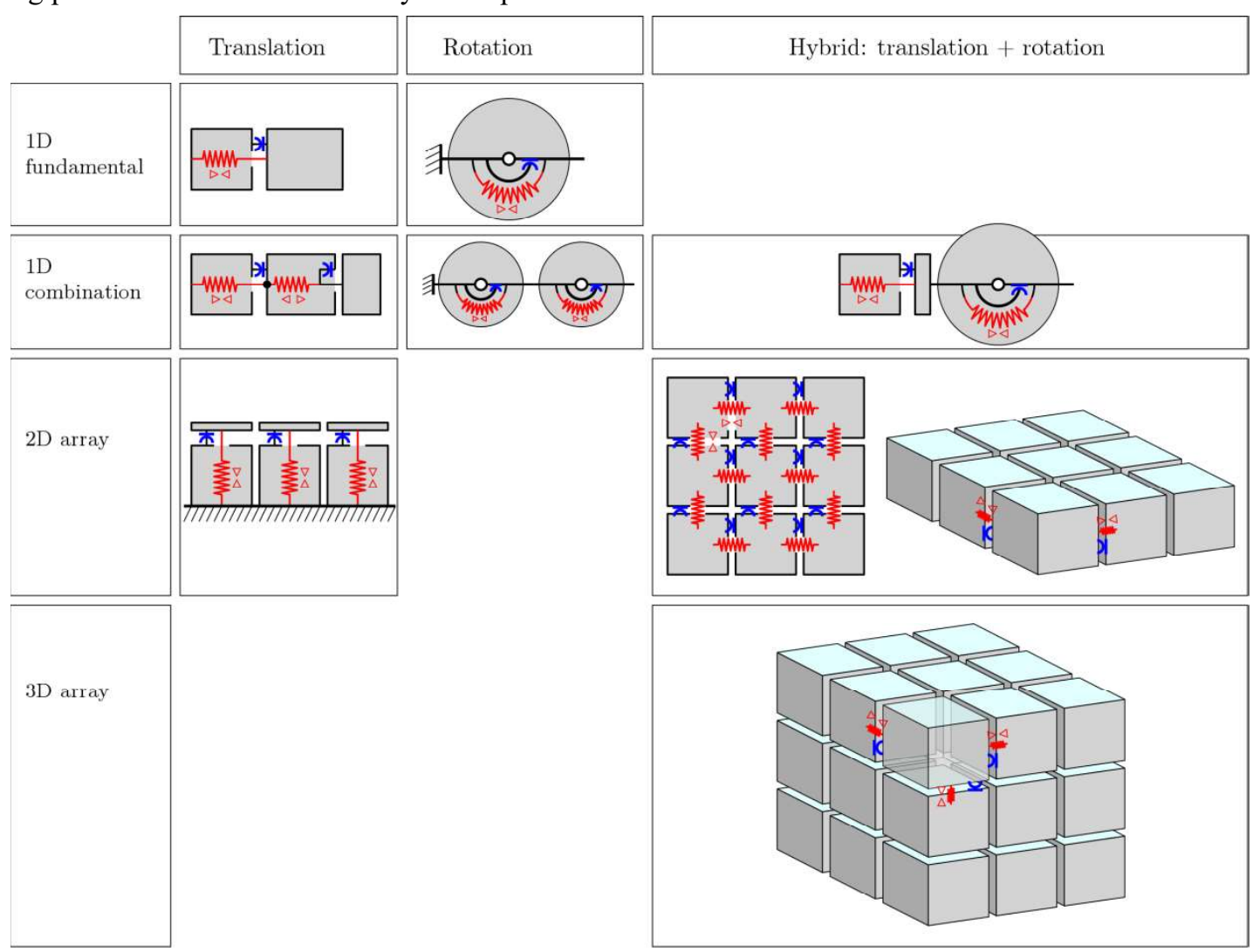

Fig. 4. Some examples of preloaded structures, from 1D element to 3D arrays. The $1^{\text {st }}$ row shows the fundamental preloaded elements: translational and rotational. Combinations of monodimensional preloaded structures are visible in the $2^{\text {nd }}$ row: a translational compression-traction solution, a 2 d.o.f. rotational one, and a combined translational-rotational structure. The $3^{\text {rd }}$ row shows planar 2D arrays of translational preloaded elements, and of combined translation-rotation elements. The last row shows a 3D translation-rotation array. 


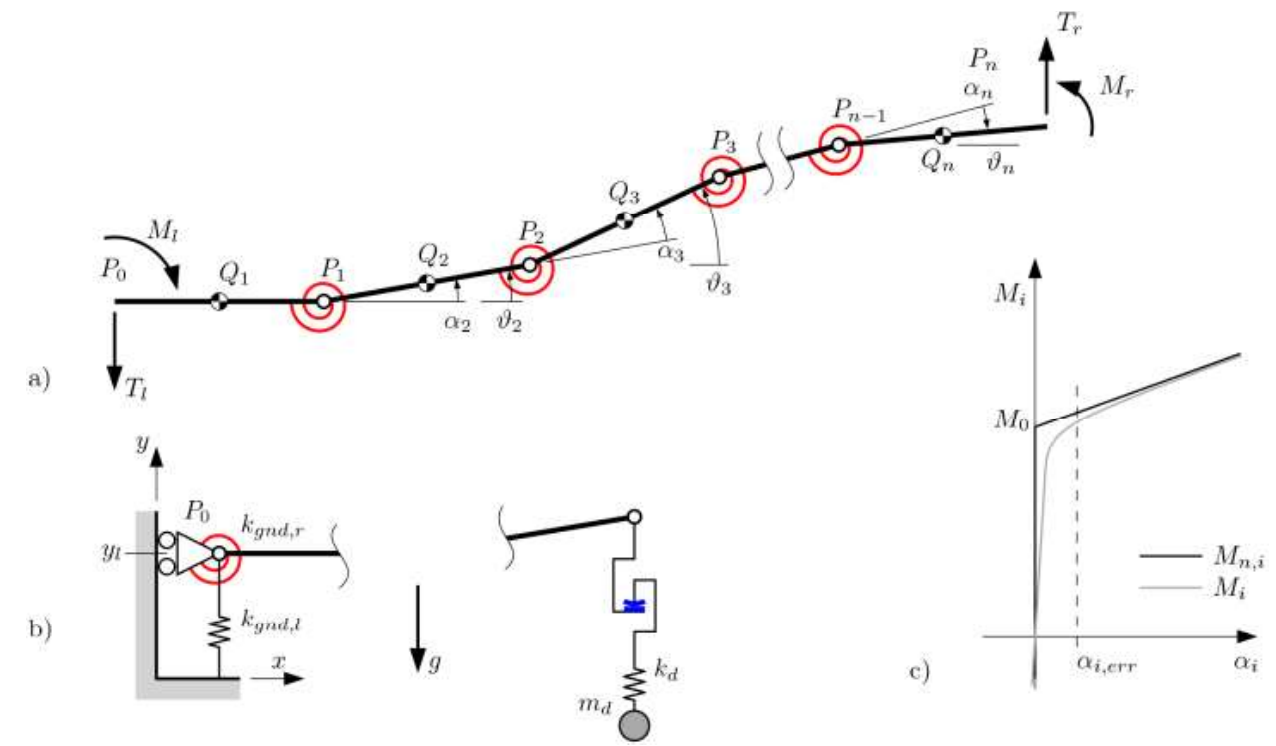

Fig. 5. Multiple D.O.F. model for a serial preloaded structure. In a) the kinematics is shown, in b) the boundary constraints and loading conditions are shown: the elastic "ground" implementation and the impactor of mass $m_{d}$; in c) the shape of the non-linear preloaded stiffness curve is shown for the general joint $i$; note that the relation is symmetric with respect to zero.

preloaded structures applied to robotics is abundant; however, it is also true that research is rather scarce where multiple-d.o.f. are concerned.

The aim of this work is precisely that of incrementing current knowledge of these complex systems, by producing a n-d.o.f. model for a preloaded structure consisting in a set of links arranged in series and connected by hinges. The layout and motion constraints for the joints are those illustrated in Fig. 3f. This kind of structure offers the following advantages with respect to a single 1 DoF preloaded structure: a) the structure has the ability to absorb the impact with an unexpected object that can occur anywhere in the structure itself; b) by having more than one preloaded elements, each potentially with a different preload level, the global deformation thresholds can be more than one.

The use-case that we choose to validate the model is that of impact energy absorption; numerical results will be compared to those emerging from an experimental campaign on a physical prototype.

\subsection{Planar N-d.o.f. Model}

Let us consider a sequence of $n$ serial links, as visible in Fig. 5a, each of length $l_{1}, \ldots, l_{n}$, mass $m_{1}, \ldots, m_{n}$, and rotational inertia $J_{1}, \ldots, J_{n}$. Let us assume, for simplicity, that each of these parameters are equal in each link: $l, m, J$. A preloaded elastic connection, such as the one illustrated in Fig. 3f, is placed between each contiguous link. The elastic connection can be modeled as a device producing a torque $M\left(\alpha_{i}\right)$, where $\alpha_{i}$ is the relative angle between the $i^{\text {th }}$ link and the next. The torque $M\left(\alpha_{i}\right)$ should follow the trend shown in the Fig. $3 \mathrm{i}$; however, since this would require discontinuities (split-field) in the elastic relation, a parametric curve was instead arbitrarily selected to approximate the nominal behaviour, as follows,

$M_{i}=\left(\frac{M_{0, i}^{2}}{\pi}\right) \operatorname{atan}\left(f_{i} \alpha_{i}\right)+k_{i} \alpha_{i}$,

where the parameter $M_{0, i}$ represents the preload torque, $k_{i}$ the stiffness of joint and $f_{i}$ is an approximation factor. This type of formulation, being continuous in $\mathbb{C}^{1}$ (as opposed to a split-field one), helps avoiding numerical noise during simulations. As will emerge from the comparison between experimental and numerical results, the simplification introduced is sufficiently appropriate to model the dynamic behaviour of the structure. The $f_{i}$ parameter can be selected by considering that the error between the actual moment $M_{n, i}$ and the approximated one $M_{i}$ is given by $\varepsilon_{r e l}=\left(M_{n, i}-M_{i}\right) / M_{n, i}$, thus it's possible to calculate $f_{i}$ corresponding to an acceptable error $\varepsilon_{\text {rel }}$ at a determined angle $\alpha_{i}$, as $f_{i}=-\tan \left(\pi\left(\varepsilon_{r e l}-1\right) / 2\right) / \alpha_{i}$. This, along with the general shape of the curve, is clearly shown in Fig. 5c.

Based on the diagram shown in Fig. 5a, the independent coordinates, in vector form, are $\boldsymbol{q}=\left\{y_{l}, \vartheta_{1}, \alpha_{1}, \ldots, \alpha_{n}\right\}$. A small-angles approximation is applied to the entire model; although a non-linear model could be developed, linearization can be desirable or even necessary where control systems need to be integrated, and where the error associated with such simplification is small. Ease of integration with a controller, especially where cobots are concerned, is an asset.

The left end-point is constrained horizontally, while it's free to move vertically.

From the principle of virtual works, it follows that: 
$\delta \Omega+\delta W_{e x t}=0$

where the first term represents the inertial forces virtual work, and the second the external forces virtual work. These can be expanded as follows,

$\delta \Omega=\sum_{i=1}^{n+1} m_{i} \ddot{y}_{i} \delta y_{i}+\sum_{i=1}^{n+1} I_{i} \ddot{\vartheta}_{i} \delta \vartheta_{i}$,

$\delta W_{\text {ext }}=-T_{l} \delta y_{l}-M_{l} \delta \vartheta_{1}+T_{r} \delta y_{r}+M_{r} \delta \vartheta_{n+1}+$ $\sum_{i=1}^{n+1} M\left(\alpha_{i}\right) \delta \alpha_{i}+\sum_{i=1}^{n+1} c_{0} \dot{\alpha}_{i} \delta \alpha_{i}+\sum_{i=1}^{n+1} m g \delta y_{i}$,

In matrix form, Eq. 3 can be written as,

$\delta \Omega=\boldsymbol{\delta} \boldsymbol{y}^{T} m \mathbf{I} \ddot{\boldsymbol{y}}+\boldsymbol{\delta} \boldsymbol{\vartheta}^{T} J \mathbf{I} \ddot{\boldsymbol{\vartheta}}$,

where $\boldsymbol{\delta} \boldsymbol{y}=\left\{\delta y_{1}, \ldots, \delta y_{n}\right\}^{T}$ and $\boldsymbol{\delta} \boldsymbol{\vartheta}=\left\{\delta \vartheta_{1}, \ldots, \delta \vartheta_{n}\right\}^{T}$ are the vector of virtual displacements, I is the identity matrix, $\ddot{\boldsymbol{y}}=\left\{\ddot{y}_{1}, \ldots, \ddot{y}_{n+1}\right\}^{T}$ and $\ddot{\boldsymbol{\vartheta}}=\left\{\ddot{\vartheta}_{1}, \ldots, \ddot{\vartheta}_{n+1}\right\}^{T}$ are the vectors of the vertical accelerations of points $y_{1}, \ldots, y_{n+1}$, and of angles $\vartheta_{1}, \ldots, \vartheta_{n+1}$ respectively. Accelerations along the longitudinal axis $\mathrm{x}$ are neglected since small vertical displacements are assumed. In order to reach a formalism based on $\boldsymbol{q}$, some changes in variables are necessary. Indeed, based on simple geometric considerations, we can write,

$$
\begin{aligned}
& \left\{\begin{array}{c}
y_{1} \\
y_{2} \\
\vdots \\
y_{n} \\
y_{n+1}
\end{array}\right\}=\left\{\begin{array}{c}
y_{l}+l / 2 \sin \vartheta_{1} \\
y_{l}+l \sin \vartheta_{1}+l / 2 \sin \vartheta_{2} \\
\vdots \\
y_{l}+l \sin \vartheta_{1}+\cdots+l / 2 \sin \vartheta_{n} \\
y_{l}+l \sin \vartheta_{1}+\cdots+l / 2 \sin \vartheta_{n+1}
\end{array}\right\} \cong \\
& {\left[\begin{array}{cccccc}
1 & l / 2 & 0 & \cdots & \cdots & 0 \\
\vdots & l & l / 2 & \ddots & & \vdots \\
\vdots & \vdots & \ddots & \ddots & \ddots & \vdots \\
\vdots & \vdots & & \ddots & l / 2 & 0 \\
1 & l & \cdots & \cdots & l & l / 2
\end{array}\right]\left\{\begin{array}{c}
y_{l} \\
\vartheta_{1} \\
\vdots \\
\vartheta_{n} \\
\vartheta_{n+1}
\end{array}\right\}=\mathbf{A}\left\{\begin{array}{c}
y_{l} \\
\vartheta_{1} \\
\vdots \\
\vartheta_{n} \\
\vartheta_{n+1}
\end{array}\right\} .(6)}
\end{aligned}
$$

It is clear that an approximation ( $\sin \vartheta_{i} \cong \vartheta_{i}$ if $\vartheta_{i}$ is small) is applied to get, from the exact formalism, to the linearized one that appears in matrix $\boldsymbol{A}$. Similarly, it can be written that,

$$
\left\{\begin{array}{c}
y_{l} \\
\vartheta_{1} \\
\vdots \\
\vartheta_{n} \\
\vartheta_{n+1}
\end{array}\right\}=\left[\begin{array}{ccccc}
1 & 0 & \cdots & \cdots & 0 \\
0 & 1 & \ddots & & \vdots \\
\vdots & \vdots & \ddots & \ddots & \vdots \\
\vdots & \vdots & & 1 & 0 \\
0 & 1 & \cdots & \cdots & 1
\end{array}\right]\left\{\begin{array}{c}
y_{l} \\
\vartheta_{1} \\
\alpha_{1} \\
\vdots \\
\alpha_{n}
\end{array}\right\}=\mathbf{B} \boldsymbol{q}
$$

It follows immediately that,

$$
\boldsymbol{y}^{T}=\mathbf{A} \mathbf{B} \boldsymbol{q}
$$

On a similar note, to write $\boldsymbol{q}$ in terms of the absolute angles $\boldsymbol{\vartheta}$, the following can be written,

$$
\left\{\begin{array}{c}
\vartheta_{1} \\
\vdots \\
\vdots \\
\vartheta_{n+1}
\end{array}\right\}=\left[\begin{array}{ccccc}
0 & 1 & 0 & \cdots & 0 \\
\vdots & \vdots & \ddots & \ddots & \vdots \\
\vdots & \vdots & & \ddots & 0 \\
0 & 1 & \cdots & \cdots & 1
\end{array}\right] \boldsymbol{q}=\mathbf{C} \boldsymbol{q}
$$

At this point, Eq. 5 can be written into the following matrix form,

$$
\begin{aligned}
\delta \Omega & =m(\mathbf{A} \mathbf{B} \boldsymbol{\delta} \boldsymbol{q})^{T} \mathbf{A} \mathbf{B} \ddot{\boldsymbol{q}}+J(\mathbf{C} \boldsymbol{\delta} \boldsymbol{q})^{T} \mathbf{C} \ddot{\boldsymbol{q}}= \\
& =\boldsymbol{\delta} \boldsymbol{q}^{T}\left(m \mathbf{B}^{T} \mathbf{A}^{T} \mathbf{A B}+J \mathbf{C}^{T} \mathbf{C}\right) \ddot{\boldsymbol{q}}
\end{aligned}
$$

Regarding the external forces and considering the situation illustrated in Fig. 5, we can write the expression in Eq. 4 as follows,

$\delta W_{\text {ext }}=$

$\left\{\delta y_{l}, \delta \vartheta_{1}, \delta y_{P, r}, \delta \vartheta_{n+1}\right\}\left\{\begin{array}{c}-T_{l} \\ -M_{l} \\ T_{r} \\ M_{r}\end{array}\right\}+$

$\left\{\delta \alpha_{1}, \ldots, \delta \alpha_{n}\right\}\left(\left\{\begin{array}{c}M\left(\alpha_{1}\right) \\ \vdots \\ M\left(\alpha_{n}\right)\end{array}\right\}+c_{o}\left\{\begin{array}{c}\dot{\alpha}_{1} \\ \vdots \\ \dot{\alpha}_{n}\end{array}\right\}\right)+$

$g\left\{\delta y_{1}, \ldots \delta y_{n}\right\}\left\{\begin{array}{c}m_{1} \\ \vdots \\ m_{n}\end{array}\right\}$

In the above equation, we call $\left\{m_{1}, \ldots, m_{n}\right\}^{T}=\boldsymbol{\mu}$ and introduce $c_{0}$ as a linear damping coefficient; in order to find the first vector in the first term on the right, we can write,

$\left\{\begin{array}{c}\delta y_{l} \\ \delta \vartheta_{1} \\ \delta y_{P, r} \\ \delta \vartheta_{n+1}\end{array}\right\}=\left[\begin{array}{cccccc}1 & 0 & 0 & \cdots & 0 & 0 \\ 0 & 1 & 0 & \cdots & 0 & 0 \\ 1 & l & l & \cdots & l & l \\ 0 & 1 & 1 & \cdots & 1 & 1\end{array}\right]$ B $\boldsymbol{\alpha} \boldsymbol{q}=\mathbf{D} \boldsymbol{\delta} \boldsymbol{q}$

Finally, if $\left\{\alpha_{1}, \ldots, \alpha_{n}\right\}^{T}=\mathbf{E} \boldsymbol{q}$, where $\mathbf{E}$ is obvious, $\boldsymbol{T}_{\text {ext }}=\left\{-T_{l},-M_{l}, T_{r}, M_{r}\right\}^{T}$, and considering Eq. 8 we can write the following matrix form for Eq. 4,

$\delta W_{\text {ext }}=(\mathbf{D} \boldsymbol{\delta} \boldsymbol{q})^{T} \boldsymbol{T}_{\text {ext }}+(\mathbf{E} \boldsymbol{\delta} \boldsymbol{q})^{T}\left(\bar{M}(\mathbf{E} \boldsymbol{q})+c_{0} \mathbf{E} \dot{\boldsymbol{q}}\right)+$

$(\mathbf{A B})^{\mathrm{T}} \boldsymbol{\mu} g=\boldsymbol{\delta} \boldsymbol{q}^{T}\left(\mathbf{D}^{T} \boldsymbol{T}_{\text {ext }}+\mathbf{E}^{T} \bar{M}(\mathbf{E} \boldsymbol{q})+c_{0} \mathbf{E}^{T} \mathbf{E} \dot{\boldsymbol{q}}\right)+$

$(\mathbf{A B})^{\mathrm{T}} \boldsymbol{\mu} g$,

where the $\bar{M}$ operator is defined as $\bar{M}(\mathbf{r})=\left\{M_{1}\left(r_{1}\right), \ldots, M_{n}\left(r_{n}\right)\right\}$, with $\boldsymbol{r}=\left\{r_{1}, \ldots, r_{n}\right\} \quad$ a general vector.

Reassembling Eq. 2 with the expressions in Eq. 10 and 11, it follows that $\left(m \mathbf{B}^{T} \mathbf{A}^{T} \mathbf{A B}+J \mathbf{C}^{T} \mathbf{C}\right) \ddot{\boldsymbol{q}}+c_{0} \mathbf{E}^{T} \mathbf{E} \dot{\mathbf{q}}+$ $\mathbf{E}^{T} \bar{M}(\mathbf{E} \boldsymbol{q})+\mathbf{D}^{T} \boldsymbol{T}_{\text {ext }}+(\mathbf{A B})^{\mathrm{T}} \boldsymbol{\mu} g=0$, thus, 
$\mathbf{H} \ddot{\boldsymbol{q}}+\mathbf{S} \dot{\boldsymbol{q}}+\mathbf{G}(\boldsymbol{q})+\mathbf{D}^{T} \boldsymbol{T}_{\text {ext }}+(\mathbf{A B})^{\mathrm{T}} \boldsymbol{\mu} g=0$,

Where $\mathbf{H}$ and $\mathbf{S}$ are the inertial coefficients matrix and the viscous damping coefficient matrix respectively; the term $\mathbf{G}(\boldsymbol{q})$ is the internal forces matrix and models the joints' elasticity. It is non-linear with respect to $\boldsymbol{q}$.

Referring again to Fig. 5a, the reader can see that the impact is simulated by using a drop mass linked to the structure at point $P_{n+1}$ with a cable of stiffness $k_{d}$; the mass of the cable is considered as integral to the weight. Upon impact, the impact mass $m_{d}$ located at coordinate $y_{m}$ produces the vertical force $F_{d}=y_{d} k_{d}$, where $y_{d}=$ $y_{m}-P_{n+1, x}$ is the elongation of the cable. This is applied to $T_{r} \in \boldsymbol{T}_{\text {ext }}$ according to the following equation,

$T_{r}=\left\{\begin{array}{cl}F_{d} & \text { if } y_{d}>0 \\ 0 & \text { if } y_{d} \leq 0\end{array}\right.$,

Fixation to the ground was implemented through an elastic linear interface consisting in a rectilinear and a rotational spring of stiffness $k_{g n d, l}$ and $k_{g n d, r}$ respectively. Finally, considering Eq. 14, the relation with $\boldsymbol{T}_{\text {ext }}$ can be represented as follows,

$\boldsymbol{T}_{\text {ext }}=\left[\begin{array}{ccccc}k_{g n d, l} & 0 & \cdots & \cdots & 0 \\ 0 & k_{g n d, r} & 0 & \cdots & 0 \\ 0 & \cdots & \cdots & \cdots & \vdots \\ 0 & \cdots & \cdots & \cdots & 0\end{array}\right] q+\left\{\begin{array}{c}0 \\ 0 \\ F_{d} \\ 0\end{array}\right\}$

In order to produce viable and stable results, the model expressed in Eq. 13 was time-integrated using the classic "RK4" Runge-Kutta method.

\subsection{Prototype}

The n-d.o.f. model presented in Section 3.1 makes certain assumptions and simplifications. It is thus interesting to test its adherence to a physical system which is slightly more complex. Indeed, the material implementation of compliant links often requires mechanisms that are considerably more complex [4], [6] than a simple elastic hinge which acts as our model captures (see Eq. 1 especially). In the following, a novel prototype is shown which is loosely based on the device described by LópezMartínez et al. [13]. As far as the kinematics is concerned, the structure itself is a 10 d.o.f. system, which takes the shape of a 6-segment compliant beam. As Fig. 6a shows, each consecutive pair of segments is connected by a hinged beam; preload between segments is applied via a spring. In the same figure, in b) and c), one can realize how the motion happens in the joint, which closely resembles the situation described in Fig. 3f. Additionally, this joint allows for small axial elongations, following the behaviour described in Fig. 3a. The joint itself behaves as the rotational joint in $\mathrm{f}$ ), in the same figure, in series with the axial one in a).

a)

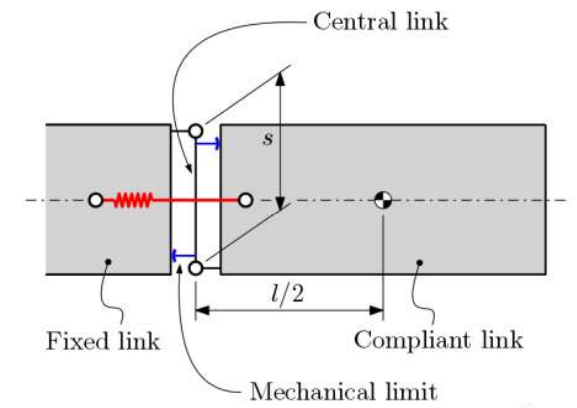

b)

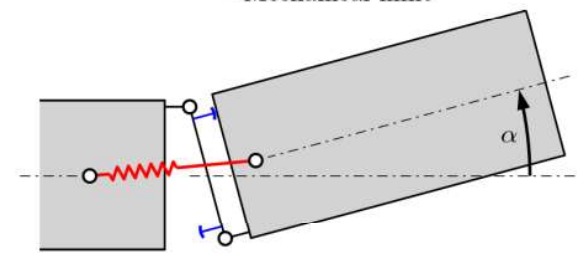

c)

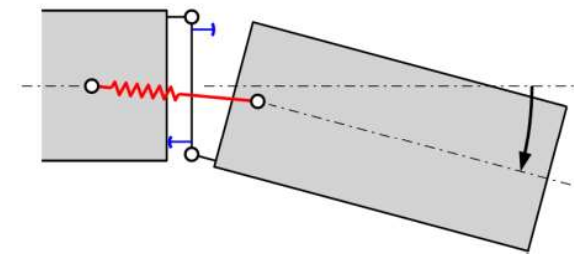

Fig. 6. Diagram of the fundamental unit of the s-Structure. In a) a general "at rest" view is shown, along with labels to the main components; in b) the upward configuration is shown, and in c) the downward one.

In Fig. 7, a 3D model is shown of the actual prototype segment along with the main components. It can be seen that the spring is placed at an angle, with a passive pulley redirecting the cable. This allows easy access to the preload screw, which is used to adjust preload. The physical prototype is shown in Fig. 9a; the structure is made of $1.5 \mathrm{~mm}$ thick carbon composite laminated sheets with glued structural junctions. The central link, as seen from Fig. 6a, was 3D printed using a PLA polymer. Polymeric low-friction bearings, coupled with polished steel shafts, were used for the joints.

It should be noted that there are some differences between the analytical model and this implementation:

i. A small-angles approximation was implemented in Eq. 6 to the $\alpha_{i}$ angles,

ii. The hinges are not centred in the midsection of the beam; in fact, based on the phase (upward or downward) the location of the axis of rotation changes,

iii. There is a dynamic asymmetry in the system, where during upward motion (Fig. 6b) the link moves with the right-hand side segment, and during downward motion (in c), same figure) it is fixed to the left-hand side segment, 


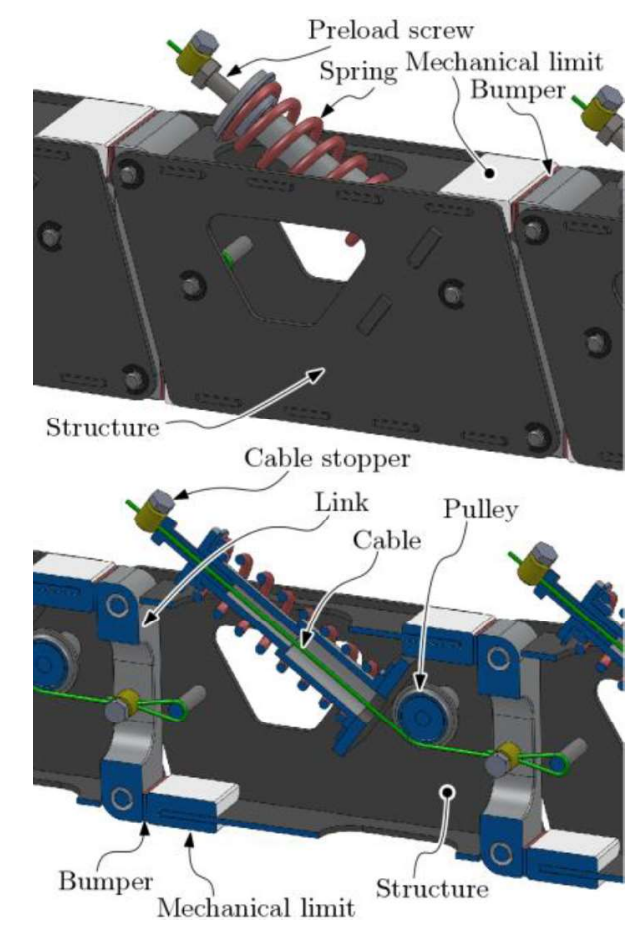

Fig. 7. Prototype segment. In a) the structure can be seen from the outside; in b) a section view is shown, with the main internal components.

iv. The torque at the hinge is generated by a linear spring in a way that introduces non-linearity,

v. The use of a pulley, as illustrated in Fig. 7, causes the cable to wound around it when the link rotates counter-clockwise and unwound when the rotation is clockwise, possibly giving rise to nonlinearities in the elastic behavior,

vi. The implementation of elastomer bumpers on the mechanical limits of the joints introduces an additional elasticity during contact.

The approximation in $\mathrm{i}$ arises from the $\sin \vartheta_{i} \cong \vartheta_{i}$, or equivalently $\sin \alpha_{i} \cong \alpha_{i}$; as such, it can be computed precisely from the simulation output. In general, the absolute error can be defined as follows,

$\varepsilon_{n}=l\left(\sum_{j=1}^{n-1}\left(\sin \vartheta_{j}-\vartheta_{j}\right)+\frac{1}{2}\left(\sin \vartheta_{n}-\vartheta_{n}\right)\right)$

Consequently, the relative error can be written as,

$\varepsilon_{n, r e l}=\frac{\varepsilon_{n}}{l\left(\sum_{j=1}^{n-1}\left(\sin \vartheta_{j}\right)+\frac{1}{2} \sin \vartheta_{n}\right)}$.

The second and third plots in Fig. 8 show the influence of this approximation in the behavior of the simulated system. It can be seen that the maximum relative error is around $1.5 \%$.

Regarding ii, in principle, the discrepancy $\varepsilon_{h}$ is proportional to the form factor $s / l$ of the segment; in fact $\varepsilon_{h} \propto s / l$. Precise evaluation of the effects on the dynamics of the system involves internal multiple contacts and impacts; it is therefore complex and ultimately out of scope for this article. As for iii, it is assumed that the influence of the link to the motion of the segments is negligible given its small mass. Regarding iv, since we assumed small angles approximation, the non-linearity on the torque generation can be considered negligible.

For point v, it can be seen from Fig. 8 that the difference in elongation of the cable with regards to the nominal value calculated for a pulley with zero diameter (or a fixed anchor point) is very small, given that during normal operation $\alpha<0.2 \mathrm{rad}$.
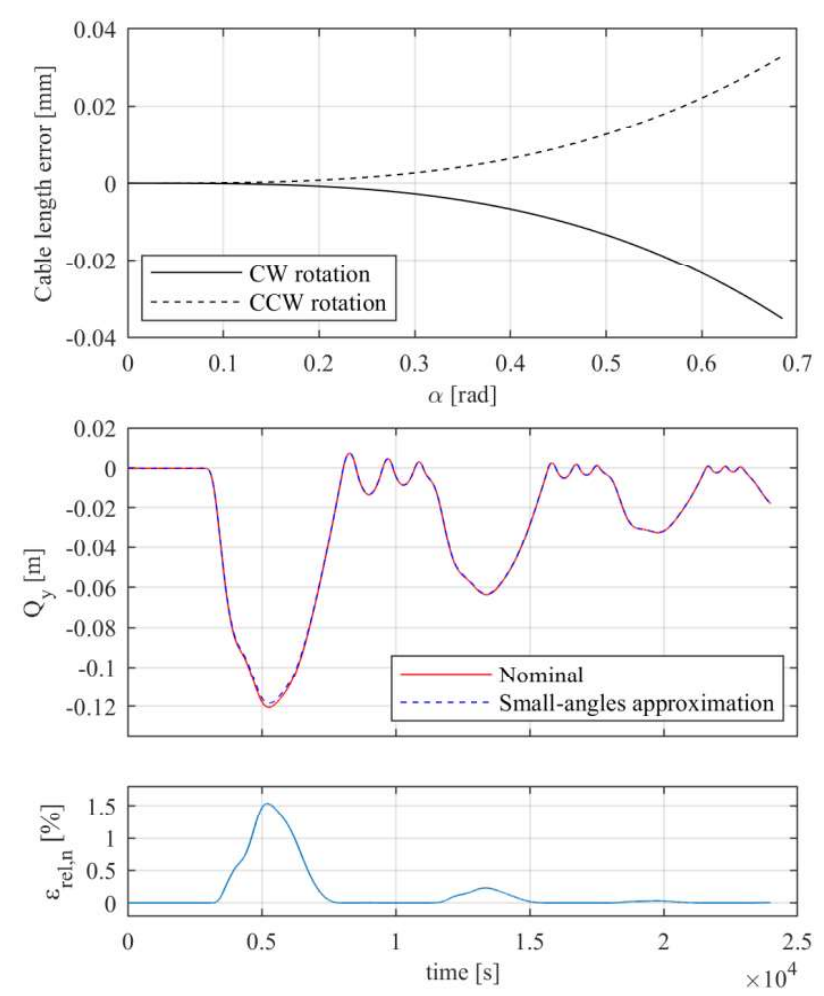

Fig. 8. Error analysis. In the first plot from the top, the cable length error due to the asymmetric effect of the pulley on the cable can be seen; in the second plot, the y-coordinate of point $Q_{n}$ is shown against time in both the nominal and small-angles approximation, whereas in the last plot the relative error $\varepsilon_{n, \text { rel }}$ can be seen.

Finally, with regards to point vi, it can be said that each elastomer bumper is characterized by a viscoelastic behavior that is active for a short period of time, namely when the bumper is in contact with the stop of the opposite link. This occurs only when $\alpha_{i}<\operatorname{arctg}\left(h_{\text {bumper }} / s\right)$, where $h_{\text {bumper }}$ is the bumper thickness. Although the dissipating effect produced by the elastomer occurs during the contact, for sake of simplicity, its global influence on the whole system has been taken into account by introducing the linear damping coefficient $c_{0}$ (Eq. 11). As far as the rubber elasticity is concerned, the impact between the bumper and the link has been assumed locally perfectly elastic; therefore kinetic energy and momentum before and after the collision are assumed to be the same: 
$\dot{\alpha}_{i}\left(t_{\text {impact }}^{+}\right)=\dot{\alpha}_{i}\left(t_{\text {impact }}^{-}\right)$, where $t_{\text {impact }}$ is the instant when the contact occurs.

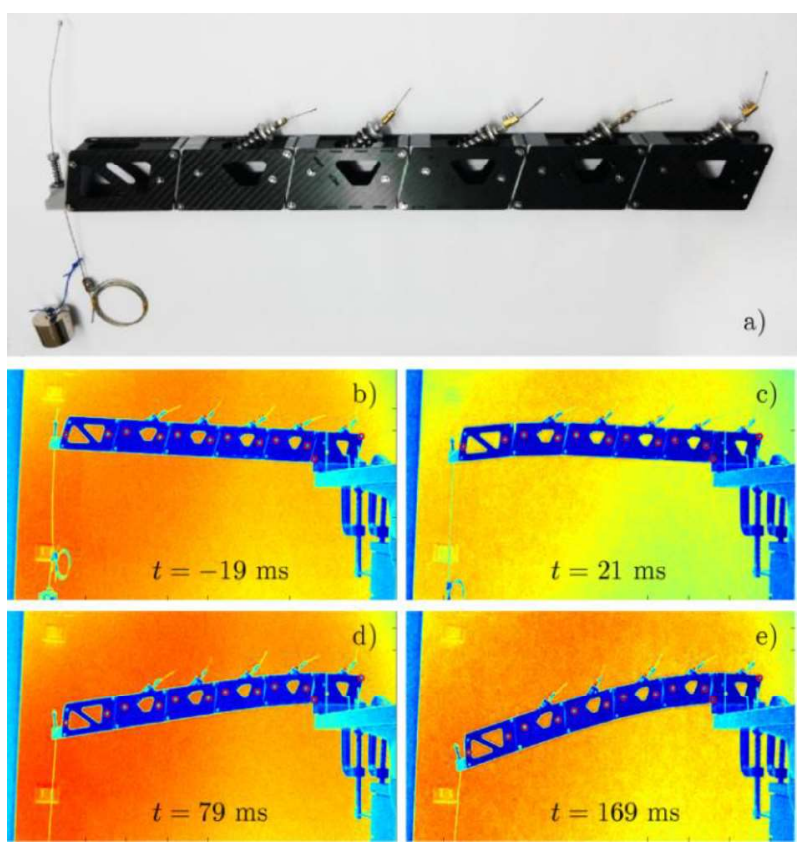

Fig. 9. Experimental prototype and capture system. The complete system is shown in a), along with the impactor and the impact spring. The reader can appreciate the different visible portion of the screws on the joints' springs, signaling different preload values. In subfigures b) to e) some snapshots of the beam are visible during the impact experiment. In this case time is referred to the instant when impact happens $(t=0 s)$.

In the experiments, the position of each segment was measured optically using a Sony RX100 IV high framerate camera and image processing software to capture the position of a set of specific points of the structure: these are circled in the snapshots in Fig. $9 b$ to e. Notice that two points are selected for each segment, in order to allow the computation of both the orientation $\vartheta_{i}$ and the position of the barycenters $Q_{i}$; the slight degree of overconstrainment allows for a more accurate measurement.
Table 1. Characterization of the stiffness and preload of the joints. The parameters of the simulation are shown: the preload moment for the $i$-th link $M_{0, i}$, the damping coefficient $c_{0}$, the elastic constant $k_{i}$, the moment of inertia $J$, the mass of each segment $m$, the impact stiffness $k_{d}$ and damping coefficient $c_{d}$, the impact mass $m_{d}$, the length $s$ of the central link and the length $l$ of each segment.

\begin{tabular}{|c|c|c|c|c|c|}
\hline Joint & 1 & 2 & 3 & 4 & 5 \\
\hline$M_{0, i}[\mathrm{Nm}]$ & 0.39 & 0.80 & 1.32 & 1.91 & 2.72 \\
\hline$c_{0}[\mathrm{Nms}]$ & & & 0.3 & & \\
\hline$k_{i}[\mathrm{Nm} / \mathrm{rad}]$ & & & 9.1875 & & \\
\hline$J\left[\mathrm{Kg} \mathrm{m}^{2}\right]$ & & & $8.167 \mathrm{E}-5$ & & \\
\hline$m[K g]$ & & & 0.098 & & \\
\hline$k_{d}[N / m]$ & & & 400 & & \\
\hline$c_{d}[\mathrm{Ns} / \mathrm{m}]$ & & & 0.5 & & \\
\hline$m_{d}[K g]$ & & & 0.220 & & \\
\hline$s[m]$ & & & 0.050 & & \\
\hline$l[m]$ & & & 0.100 & & \\
\hline
\end{tabular}

In order to provide validation to the analytical model described in Section 3.1, by using the experimental prototype, the parameters of the latter are to be correctly identified, especially the preload on the spring. Therefore, the prototype was measured, having care of directly characterizing the effective preload applied to each joint. Considering the geometry highlighted in Fig. 6, the stiffness $k_{i}$ of the joints was computed from the springs nominal coefficient of elasticity $(14.7 \mathrm{~N} / \mathrm{mm})$. These data along with the other simulation parameters are summarized in Table 1.

\subsection{Results and discussion}

Implementation of the analytical model into a numerical RK4 time integration engine produced the results shown in Fig. 11 for the static case, and those illustrated Fig. 10 for the dynamical behavior.

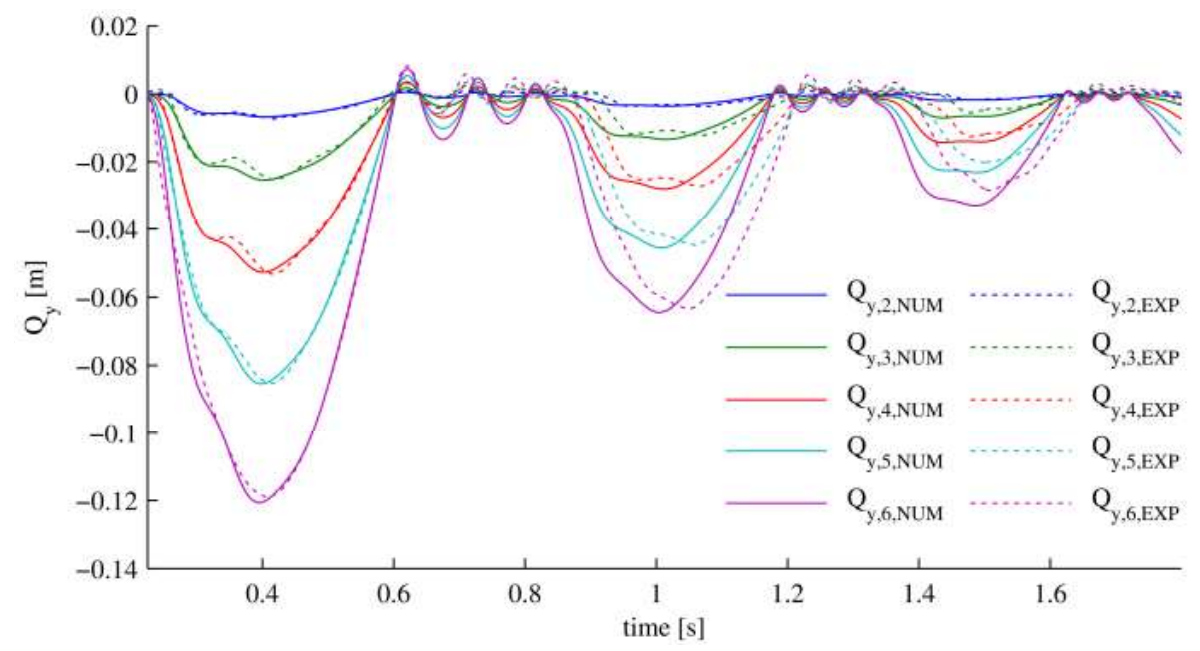

Fig. 10. Coordinate y of the barycentre $Q_{i}$ of the links over time following impact at $t=0.225 \mathrm{~s}$ of the impactor. A comparison between results of the numerical (solid line, $\Delta t=10^{-5}$ ) and experimental (dashed line, $\Delta t=10^{-3}$ ) runs is shown. 


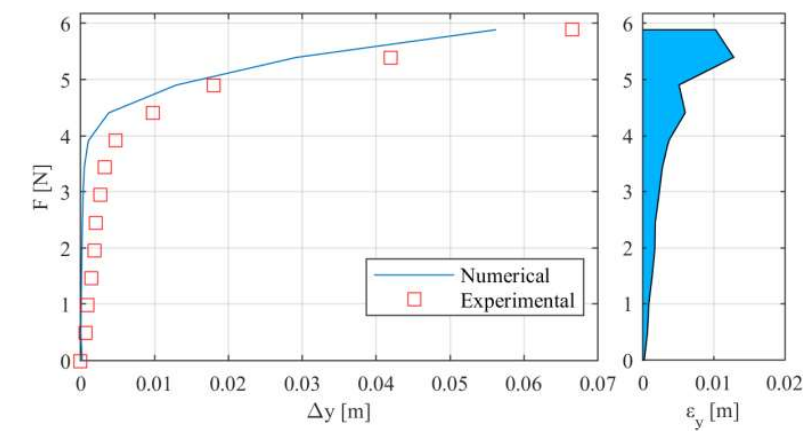

Fig. 11. Static stiffness plot of the beam. The displacement $\Delta y$ is measured at point $P_{6}$ in the model and at the corresponding point in the prototype. On the left, the numerical and experimental curves are shown, while on the right the displacement error is reported.

Experimental results are also shown in the same figures, so as to provide a good understanding of the adherence of the model to reality. In Fig. 11, a comparison of the results show similar behavior, in which the main reason for error is due to a certain deviation happening in the steep part of the plot; this is very likely due to the flexibility of the beam given by the rubber bumpers (see Fig. 7). The data in Fig. 10 show the coordinate $y$ of the geometric center $Q_{i}$ for link $i=2, \ldots, 6$. Link 1 was intentionally left out since experimental noise was larger than the effective motion of the observed markers through the experiment. Ultimately, the position (and orientation) is fixed to the frame in both contexts, and is thus uninteresting for our purpose.

Results show impact happening at approximately $t=$ $0.225 \mathrm{~s}$; primary and secondary oscillations are visible in all modules. While the former is due to the storage of most of the kinetic energy of the impactor mass, and is thus larger, the latter is due to the oscillation of the impact spring-mass system and possibly to the relative motion of the segments, which are, incidentally, of lower energetic content. The first zero-crossing for the numerical curves shows remarkable time-coherence $(0.23 \%$ on average) with the experimental data, as does the total displacement of all links $(0.05 \%$, averaged); it is worth noting that numerical and experimental noise do not allow a precise measurement of these errors, so they must be taken as indicative. Furthermore, the numerical results show a noticeable time-shift (leading). This is possibly due to friction which manifests at the interface between the impactor cable and its guide-conduit. Furthermore, the interval $t=0.65 \div 0.85 \mathrm{~s}$ shows some difference in the model's representation, and is likely due to the shifting of the relevant rotation axis during upward versus downward motion, as highlighted at the end of Section 3.2; this effect is not completely described by our model, especially the energy-loss due to internal impacts and contacts.
To provide a more comprehensive validation of the model, it is perhaps useful to examine the error between the numerical and the experimental curves, defined as follows,

$\varepsilon_{G y, i}=\frac{\left|G_{y, i, N U M}-G_{y, i, E X P}\right|}{\max \left(\left|G_{y, i, E X P}\right|\right)}$.

In Fig. 12, the error $\varepsilon_{G y, i}$ is shown for links $i=2, \ldots, 6$. It should be noted that in these charts only show the error during the first impact is shown, that is, between $t=$ $0.225 s$ and $0.650 s$. The rationale for this choice is that the time-shift, which is described in the previous paragraph, does not allow for a rigorous comparison.

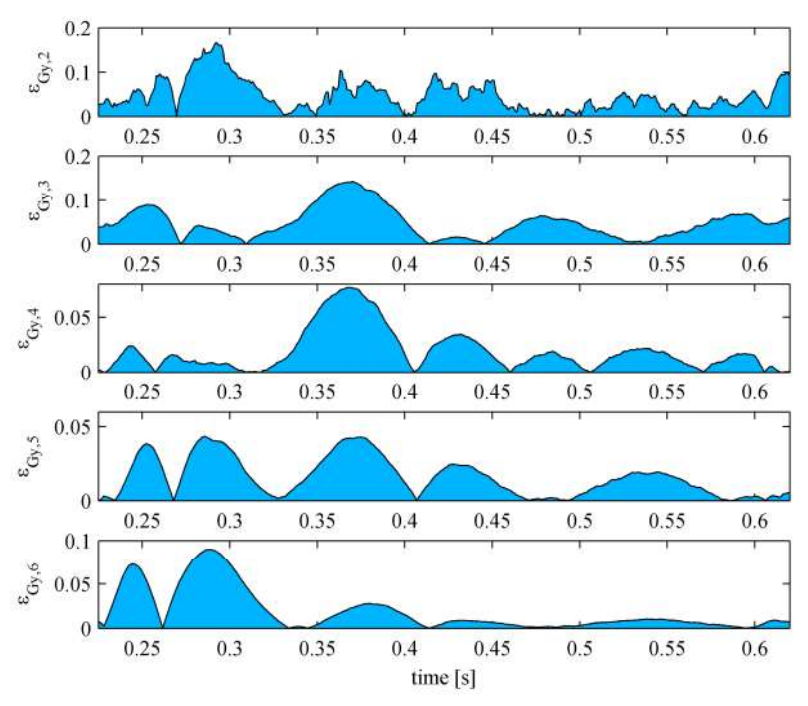

Fig. 12. Relative error between experimental and numerical results for the 5 links; the values are relative to the maximum absolute $Q_{y, i}$ of each link's experimental result. Error values are bound to the $0-1$ interval.

As shown in Fig. 12, the error remains well below 20\% for links 2 and $3\left(\varepsilon_{G y, 2}<0.18, \varepsilon_{G y, 3}<0.16\right)$, below $10 \%$ for links 5 and $6\left(\varepsilon_{G y, 5}<0.05, \varepsilon_{G y, 6}<0.09\right)$ and below $5 \%$ for link $4\left(\varepsilon_{G y, 4}<0.08\right)$. The fact that gross total displacement is as low as $0.05 \%$, while at the same time point-by-point error can be as high as $20 \%$ indicates that while primary high-energy displacements are very well represented by the model, secondary low-energy motions are not. This is expected, given that the latter are mainly due to internal contacts and impacts which are not directly modeled.

\section{CONCLUSION}

In the field of collaborative robotics, impact mitigation is a core issue. This paper proposed a framework for the implementation of $\mathrm{n}$-dof preloaded mechanisms as the frame of cobots for passive collision protection.

In Section 1, an in-depth analysis of the state-of-the-art was performed, with special interest in the application of 
preload in the field of robotics. In Section 2 the framework was presented, defining the fundamental elements of a preloaded system and providing some examples as to some possible architectures. In Section 3, fundamental rotational preloaded elements were implemented in the model of an n-d.o.f. serial structure. A prototype was built to validate the analytical model through an impact test, which was performed both on the model - numerically and on the prototype. Results have shown good adherence between the model and the experimental results, with a $0.23 \%$ time-coherence and $0.05 \%$ maximum total displacement error. First impact shows point-by-point error values below $20 \%$ and as low as $4 \%$ for the entire relevant acquisition.

The framework which was explored and presented in this work provides a good foundation for an organic study of preloaded structures employed in collaborative robotics. The n-dof model designed to describe the behaviour of an illustrative example of these systems showed remarkable accuracy in high-energy oscillation upon impact.

Taking advantage from the impact-mitigation traits of these systems, some directions of future development concern the application of the framework to the field of mobile robots, as well as that of impact-resistant structures. Also, we foresee the implementation of multidimensional n-DoF preloaded structures to smaller scales, whereby novel materials with peculiar mechanical properties (such as nonlinear reversible elasticity or high impact resistance) can be designed within the framework of the so-called architected materials [32], [33], and fabricated even at the nanometric scale exploiting the rapidly evolving technologies for advanced manufacturing [33] or, specifically, additive manufacturing [33], [34].

\section{BIBLIOGRAPHY}

[1] Colgate, J., Wannasuphoprasit, W. and Peshkin, M. A., 1996, "Cobots: robots for collaboration with human operators", Proceedings of the ASME Dynamic Systems and Control Division, vol. 58, pp.433-439.

[2] Yamada, Y., Hirasawa, Y., Huang, S., Umetani, Y. and Suita K., 1997, "Human-robot contact in the safeguarding space," IEEE/ASME Transactions on Mechatronics, 2(4), pp. 230-236.

[3] Lim, H.-O. and Tanie, K., 2000, "Passive viscoelastic trunk and passively movable base," International Journal of Robotics Research, 19(4), pp. 307-335.

[4] Seong-Sik, Y., Sungchul, K., Seung-kook, Y., Seung-Jong, K., Young-Hwan, K. and Munsang, K., 2005, "Safe Arm Design with MR-based Passive Compliant Joints and Visco-elastic Covering for Service Robot Applications," Journal of Mechanical Science and Technology, 19(10), pp. 1835-1845.

[5] Park, J.-J., Kim, B.-S., Song, J.-B. and Kim, H.-S., 2008, "Safe link mechanism based on nonlinear stiffness for collision safety," Mechanism and Machine Theory, 43(10), pp. 13321348.

[6] Park, J.-J., Kim, H.-S. and Song, J.-B., 2009, "Safe Robot Arm with Safe Joint Mechanism using Nonlinear Spring System for
Collision Safety," 2009 IEEE International Conference on Robotics and Automation, Kobe, Japan.

[7] Park, J.-J. and Song, J.-B., 2010, "A Nonlinear Stiffness Safe Joint Mechanism Design for Human Robot Interaction," Journal of Mechanical Design, 132

[8] Park, J.-J. and Song, J.-B., 2010, "Safe Joint Mechanism using Inclined Link with Springs for Collision Safety and Positioning Accuracy of a Robot Arm," 2010 IEEE International Conference on Robotics and Automation, Anchorage, Alaska, USA.

[9] López-Martínez, J., Blanco-Claraco, J. L., García-Vallejo, D. and Giménez-Fernández, A., 2015, "Design and analysis of a flexible linkage for robot safe operation in collaborative scenarios," Mechanism and Machine Theory, 92, pp. 1-16.

[10] Medina, J., Lozano, P., Jardòn, A. and Balaguer, C., 2016 "Design and Characterization of a Novel Mechanism of Multiple Joint," IEEE/RSJ International Conference on Intelligent Robots and Systems (IROS), Daejeon, Korea.

[11] Lauzier, N. and Gosselin, C., 2015, "A Comparison of the Effectiveness of Design Approaches for HumanFriendly Robots,"Journal of Mechanical Design, 137.

[12] Park, J.-J., Song, J.-B. and Haddadin, S., 2015, "Collision analysis and safety evaluation using a collision model for the frontal robot-human impact," Robotica, 33, pp. 1536-1550.

[13] López-Martínez, J., García-Vallejo, D., Giménez-Fernández, A. and Torres-Moreno, J., 2014, "A Flexible Multibody Model of a Safety Robot Arm for Experimental Validation and Analysis of Design Parameters," Journal of Computational and Nonlinear Dynamics, 9.

[14] Courreges, F., Laribi, M. A., Arsicault, M. and Zeghloul, S., 2016, "Designing a biomimetic model of non-linear elastic safety mechanism for collaborative robots," 2016 IEEE 14th International Conference on Industrial Informatics (INDIN), Poitiers, France.

[15] Wolf, S. and Hirzinger, G., 2008, "A new variable stiffness design: Matching requirements of the next robot generation," in 2008 IEEE International Conference on Robotics and Automation, Pasadena, CA, USA.

[16] Tonietti, G., Schiavi, R. and Bicchi, A., 2005, "Design and Control of a Variable Stiffness Actuator for Safe and Fast Physical Human/Robot Interaction," Proceedings of the 2005 IEEE International Conference on Robotics and Automation, Barcelona, Spain.

[17] Mathijssen, G., Brackx, B., Damme, M. V., Lefeber, D. and Vanderborght, B., 2013, "Series-parallel elastic actuation (SPEA) with intermittent mechanism for reduced motor torque and increased efficiency," 2013 IEEE/RSJ International Conference on Intelligent Robots, Tokyo, Japan.

[18] Bely, P. Y., 2003 "The Design and Construction of Large Optical Telescopes," Astronomy and Astrophysics Library, Springer-Verlag.

[19] Olivieri, L., Antonello, A., Savioli, L. and Francesconi, A., 2014, "Dynamic behavior of a semi-androgynous small satellite docking interface," in 65th International Astronautical Congress, Toronto, Canada.

[20] Wu, Y.-S. and Lan, C.-C., 2014, "Linear variable-stiffness mechanisms based on preloaded curved beams," Journal of Mechanical Design, 136.

[21] Qin, Z., Yan, S. and Chu, F., 2010, "Dynamic analysis of clamp band joint system subjected to axial vibration," Journal of Sound and Vibration, 329, pp. 4486-4500.

[22] Liguori, C., Paciello, V., Paolillo, A., Pietrosanto, A. and Sommella, P., 2013, "Characterization of motorcycle suspension systems: Comfort and handling performance evaluation," 2013 IEEE International Instrumentation and Measurement Technology Conference (I2MTC), Minneapolis, MN, USA. 
[23] Baronti, F., Lenzi, F., Roncella, R., Saletti, R. and Di Tanna, O., 2007, "Embedded Electronic Control System for Continuous Self-Tuning of Motorcycle Suspension Preload," in 2007 Mediterranean Conference on Control and Automation, Athens, Greece.

[24] Nirmal, M. D., Mandal, K. and Sun, Y. Q., 2014, "Impact forces at dipped rail joints," Journal of Rail and Rapid Transit, 230(1), pp. 271-282.

[25] Pashkevich, A., Klimchik, A. and Chablat, D., 2011, "Enhanced stiffness modeling of manipulators with passive joints," Mechanism and Machine Theory, 46, pp. 662-679.

[26] Dwivedy, S. K. and Eberhard, P., 2006, "Dynamic analysis of flexible manipulators, a literature review," Mechanism and Machine Theory, vol. 41, pp. 749-777.

[27] Shabana, A.A., 1997, "Flexible multibody dynamics: Review of past and recent developments," Multibody System Dynamics, 1(2), pp. 189-222.

[28] Bauchau, O. A., 2011, "Flexible Multibody Dynamics", Springer Netherlands.

[29] Boscariol, P., Gallina, P., Gasparetto, A., Giovagnoni, M., Scalera, L. and Vidoni, R., 2017, "Evolution of a dynamic model for flexible multibody systems," in Advances in Italian Mechanism Science, Springer, pp. 533-541.

[30] Vanderborght, B., Albu-Schaeffer, A., Bicchi, A., Burdet, E., Caldwell, D., Carloni, R., Catalano, M., Eiberger, O., Friedl, W., Ganesh, G., Garabini, M., Grebenstein, M., Grioli, G., Haddadin, S., Hoppner, H., Jafari, A., Laffranchi, M., Lefeber, D., Petit, F. and Strami, S., 2013, "Variable impedance actuators: A review," Robotics and Autonomous Systems, 61(12), pp. 1601-1614.

[31] Lauzier, N. and Gosselin, C., 2011, "Series clutch actuators for safe physical human-robot interaction," in IEEE International Conference on Robotics and Automation (ICRA), Shanghai, China.

[32] Schaedler, T. A. and Carter, W. B., 2016, "Architected Cellular Materials," Annual Review of Materials Research, 46(1), pp. 187-210.

[33] Bauer, J., Meza, L. R., Schaedler, T. A., Schwaiger, R., Zheng, X. and Valdevit, L., 2017, "Nanolattices: An Emerging Class of Mechanical Metamaterials," Advanced Materials, 29(40), p. 1701850 .

[34] Valdevit L., 2016, "3D manufacturing of micro and nanoarchitected materials," Proceedings SPIE 9738, Laser 3D Manufacturing III, San Francisco, California, USA. 